\title{
A THERMOELASTIC CRACK PROBLEM FOR AN ANISOTROPIC SLAB
}

\author{
D. L. CLEMENTS and T. R. TAUCHERT
}

(Received 16 June 1978)

(Revised 15 November 1978)

\begin{abstract}
The problem of determining the temperature, displacement and stress fields around a single crack in an anisotropic slab is considered. The problem is reduced to Fredholm integral equations which may be solved numerically.
\end{abstract}

\section{Introduction}

It is now widely accepted that the behaviour of some anisotropic materials closely models the behaviour of certain fibre-reinforced composites (see, for example, Spencer [10]). This has caused a renewed interest in anisotropic materials in recent years, and a number of new solutions to boundary-value problems have been obtained. Specifically, Clements $[4,5]$ has presented solutions to problems involving cracks in anisotropic slabs and anisotropic layered materials while Tauchert and Aköz [13], Aköz and Tauchert [1], Clements [6], Clements and Toy [8], Atkinson and Clements [2] and Chang [3] have solved various thermostatic and thermoelastic problems for anisotropic materials. References to various other works in this area may be found in the review article by Tauchert [12].

In the present paper the problem of determining the temperature, displacement and stress fields around a crack in an anisotropic slab is considered. The bounding planes of the slab are subjected to an arbitrary temperature distribution and arbitrary tractions. Over the crack the heat flux and the tractions are prescribed. For these boundary conditions the problem is reduced to a number of Fredholm integral equations which may be solved numerically. Numerical results are obtained for a particular transversely isotropic material and the results are used to make some qualitative comments about the nature of the stress near the crack tip. 


\section{Statement of problem and basic equations}

Take Cartesian coordinates $x_{1}, x_{2}, x_{3}$ in a homogeneous anisotropic elastic slab. Contained in the slab is a crack in the region $x_{2}=0,\left|x_{1}\right|<a,-\infty<x_{3}<\infty$ (Fig. 1). On the faces of the crack the heat flux and the tractions are prescribed. On the faces of the slab at $x_{2}= \pm h$ the temperature and tractions are prescribed. It is required to find the displacement and stress fields throughout the slab.

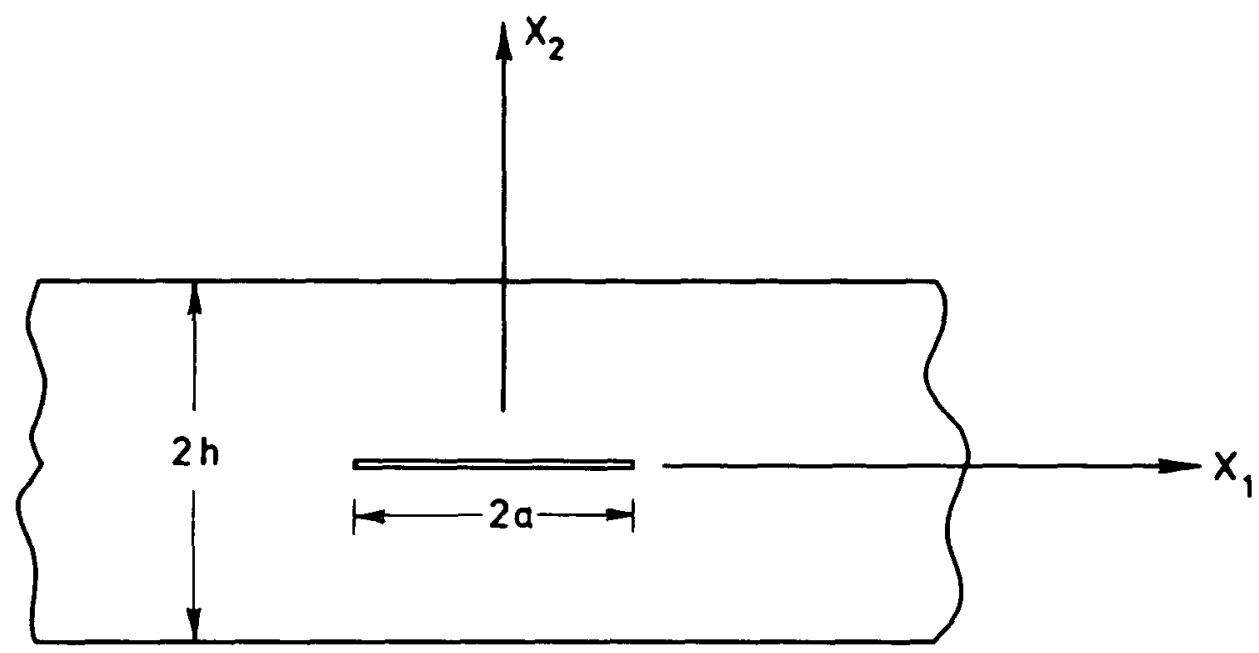

Fig. 1. Location of the crack in the slab.

In Clements [6] some representations are derived for the temperature $T$, displacement $u_{k}$ and stress $\sigma_{i j}$ in an anisotropic material. These representations take the form

$$
\begin{aligned}
T & =\frac{1}{\pi} \mathscr{R} \int_{0}^{\infty} A(p) \exp \left(i p z^{\prime}\right) d p, \\
u_{k} & =\frac{1}{\pi} \mathscr{R} \int_{0}^{\infty}\left\{\sum_{\alpha} A_{k \alpha} E_{\alpha}(p) \exp \left(i p z_{\alpha}\right)+C_{k} A(p) p^{-1} \exp \left(i p z^{\prime}\right)\right\} d p, \\
\sigma_{i j} & =\frac{1}{\pi} \mathscr{R} \int_{0}^{\infty}\left\{\sum_{\alpha} L_{i j \alpha} E_{\alpha}(p) i p \exp \left(i p z_{\alpha}\right)+\left(i N_{i j}-\beta_{i j}\right) A(p) \exp \left(i p z^{\prime}\right)\right\} d p,
\end{aligned}
$$

where $\mathscr{R}$ denotes the real part of a complex number and $A(p)$ and $E_{\alpha}(p), \alpha=1,2,3$, are functions to be determined from boundary conditions. Also, in (2.1)-(2.3) the constants $\tau$ (in $z^{\prime}=x_{1}+\tau x_{2}$ ), $p_{\alpha}$ (in $z_{\alpha}=x_{1}+p_{\alpha} x_{2}$ ), $A_{k \alpha}, C_{k}, L_{i j \alpha}$ and $N_{i j}$ are related to the coefficients of heat conduction $\lambda_{i j}$, the elastic constants $c_{i j k l}$, and the stress-temperature coefficients $\beta_{i j}$ in a way which is indicated in Clements [6]. 
At this point it is appropriate to note that the repeated suffix summation convention (summing from 1 to 3) will be used throughout the paper for Latin suffices only. Summation over Greek suffices (also from 1 to 3 ) will always be indicated explicitly.

The representations (2.1)-(2.3) tend to zero as $x_{2} \rightarrow \infty$. The corresponding expressions for $T, u_{k}$ and $\sigma_{i j}$ which tend to zero as $x_{2} \rightarrow-\infty$ will also be useful in the subsequent analysis. They may be derived by following the procedure used in Clements [6] and take the form

$$
\begin{aligned}
T & =\frac{1}{\pi} \mathscr{R} \int_{0}^{\infty} A(p) \exp \left(-i p z^{\prime}\right) d p \\
u_{k} & =\frac{1}{\pi} \mathscr{R} \int_{0}^{\infty}\left\{\sum_{\alpha} A_{k \alpha} E_{\alpha}(p) \exp \left(-i p z_{\alpha}\right)-C_{k} A(p) p^{-1} \exp \left(-i p z^{\prime}\right)\right\} d p \\
\sigma_{i j} & =-\frac{1}{\pi} \mathscr{R} \int_{0}^{\infty}\left\{\sum_{\alpha} L_{i j \alpha} E_{\alpha}(p) i p \exp \left(-i p z_{\alpha}\right)-\left(i N_{i j}-\beta_{i j}\right) A(p) \exp \left(-i p z^{\prime}\right)\right\} d p
\end{aligned}
$$

where, as in (2.1)-(2.3), the $A(p)$ and $E_{\alpha}(p), \alpha=1,2,3$, are unknown functions which will be determined from the boundary conditions. The constants in (2.4)(2.6) are the same as those in (2.1)-(2.3).

For the purposes of the present paper, it is convenient to write the temperature, displacement and stress as the sum of three fields. Specifically, we write

$$
\begin{gathered}
T=T^{(1)}+T^{(2)}+T^{(3)}, \\
u_{k}=u_{k}^{(1)}+u_{k}^{(2)}+u_{k}^{(3)}, \\
\sigma_{i j}=\sigma_{i j}^{(1)}+\sigma_{i j}^{(2)}+\sigma_{i j}^{(3)},
\end{gathered}
$$

where, from (2.1)-(2.3),

$$
\begin{aligned}
& T^{(1)}=\frac{1}{\pi} \mathscr{R} \int_{0}^{\infty} A^{(1)}(p) \exp \left(i p z^{\prime}\right) d p \\
& u_{k}^{(1)}=\frac{1}{\pi} \mathscr{R} \int_{0}^{\infty}\left\{\sum_{\alpha} A_{k \alpha} E_{\alpha}^{(1)}(p) \exp \left(i p z_{\alpha}\right)+C_{k} A^{(1)}(p) p^{-1} \exp \left(i p z^{\prime}\right)\right\} d p \\
& \sigma_{i j}^{(1)}=\frac{1}{\pi} \mathscr{R} \int_{0}^{\infty}\left\{\sum_{\alpha} L_{i j \alpha} E_{\alpha}^{(1)}(p) i p \exp \left(i p z_{\alpha}\right)+\left(i N_{i j}-\beta_{i j}\right) A^{(1)}(p) \exp \left(i p z^{\prime}\right)\right\} d p
\end{aligned}
$$


and, from (2.4)-(2.6),

$$
\begin{aligned}
T^{(2)} & =\frac{1}{\pi} \mathscr{R} \int_{0}^{\infty} A^{(2)}(p) \exp \left(-i p z^{\prime}\right) d p \\
u_{k}^{(2)} & =\frac{1}{\pi} \mathscr{R} \int_{0}^{\infty}\left\{\sum_{\alpha} A_{k \alpha} E_{\alpha}^{(2)}(p) \exp \left(-i p z_{\alpha}\right)-C_{k} A^{(2)}(p) p^{-1} \exp \left(-i p z^{\prime}\right)\right\} d p \\
\sigma_{i j}^{(2)} & =-\frac{1}{\pi} \mathscr{R} \int_{0}^{\infty}\left\{\sum_{\alpha} L_{i j \alpha} E_{\alpha}^{(2)}(p) i p \exp \left(-i p z_{\alpha}\right)-\left(i N_{i j}-\beta_{i j}\right) A^{(2)}(p) \exp \left(-i p z^{\prime}\right)\right\} d p
\end{aligned}
$$

For $T^{(3)}, u_{k}^{(3)}$ and $\sigma_{i j}^{(3)}$ we consider the regions $0<x_{2}<h$ and $-h<x_{2}<0$ separately. Hence

$$
\begin{aligned}
& T^{(3)}=\frac{1}{\pi} \mathscr{R} \int_{0}^{\infty} A^{+}(p) \exp \left(i p z^{\prime}\right) d p \quad \text { for } 0<x_{2}<h, \\
& u_{k}^{(3)}=\frac{1}{\pi} \mathscr{R} \int_{0}^{\infty}\left\{\sum_{\alpha} A_{k \alpha} E_{\alpha}^{+}(p) \exp \left(i p z_{\alpha}\right)+C_{k} A^{+}(p) p^{-1} \exp \left(i p z^{\prime}\right)\right\} d p \\
& \text { for } 0<x_{2}<h \\
& \sigma_{i j}^{(3)}=\frac{1}{\pi} \mathscr{R} \int_{0}^{\infty}\left\{\sum_{\alpha} L_{i j \alpha} E_{\alpha}^{+}(p) i p \exp \left(i p z_{\alpha}\right)+\left(i N_{i j}-\beta_{i j}\right) A^{+}(p) \exp \left(i p z^{\prime}\right)\right\} d p \\
& \quad \text { for } 0<x_{2}<h \\
& T^{(3)}=\frac{1}{\pi} \mathscr{R} \int_{0}^{\infty} A^{-}(p) \exp \left(-i p z^{\prime}\right) d p \quad \text { for }-h<x_{2}<0, \\
& u_{k}^{(3)}=\frac{1}{\pi} \mathscr{R} \int_{0}^{\infty}\left\{\sum_{\alpha} A_{k \alpha \alpha} E_{\alpha}^{-}(p) \exp \left(-i p z_{\alpha}\right)-C_{k} A^{-}(p) p^{-1} \exp \left(-i p z^{\prime}\right)\right\} d p \\
& \quad \text { for }-h<x_{2}<0 \\
& \sigma_{i j}^{(3)}=-\frac{1}{\pi} \mathscr{R} \int_{0}^{\infty}\left\{\sum_{\alpha} L_{i j \alpha} E_{\alpha}^{-}(p) i p \exp \left(-i p z_{\alpha}\right)-\left(i N_{i j}-\beta_{i j}\right) A^{-}(p) \exp \left(-i p z^{\prime}\right)\right\} d p \\
& \text { for }-h<x_{2}<0 .
\end{aligned}
$$

\section{Temperature field}

On $x_{2}=0,\left|x_{1}\right|<a$ the heat flux is prescribed so that the boundary condition in this region is

$$
\lambda_{12} \frac{\partial T}{\partial x_{1}}+\lambda_{22} \frac{\partial T}{\partial x_{2}}=-f\left(x_{1}\right)
$$

Also, the temperature $T$ is prescribed on the faces $x_{2}= \pm h$ of the slab so that

$$
T=g_{1}\left(x_{1}\right) \text { on } x_{2}=h
$$


and

$$
T=g_{2}\left(x_{1}\right) \text { on } x_{2}=-h
$$

where $g_{1}$ and $g_{2}$ are given. We wish to find the temperature field throughout the slab.

A solution to this problem has been obtained by Clements and Tauchert [7]. The temperature is given by (2.7), (2.10), (2.13), (2.15) and (2.19) with

$$
\begin{aligned}
& A^{+}(p)=\left(\lambda_{12}+\lambda_{22} \tau\right)^{-1} A(p), \\
& A^{-}(p)=\left(\lambda_{12}+\lambda_{22} \bar{\tau}\right)^{-1} A(p), \\
& A^{(1)}(p)=Q(p) A(p)+U(p), \\
& A^{(2)}(p)=Q(p) A(p)+V(p),
\end{aligned}
$$

where the bar denotes the complex conjugate and the functions $Q(p), U(p)$, $V(p)$ are given by

$$
\begin{aligned}
& Q(p)=-\left\{\left(\lambda_{12}+\lambda_{22} \tau\right)^{-1} \exp [i p(\tau-\bar{\tau}) h]-\left(\lambda_{12}+\lambda_{22} \bar{\tau}\right)^{-1}\right\} D, \\
& U(p)=\left[B_{1}(p) \exp (-i p \bar{\tau} h)-B_{2}(p) \exp (i p \bar{\tau} h)\right] D, \\
& V(p)=\left[-\bar{B}_{1}(p) \exp (i p \bar{\tau} h)+\bar{B}_{2}(p) \exp (-i p \bar{\tau} h)\right] D
\end{aligned}
$$

with

$$
D=\{\exp [i p(\tau-\bar{\tau}) h]-\exp [-i p(\tau-\bar{\tau}) h]\}^{-1}
$$

and $B_{1}(p)$ and $B_{2}(p)$ may be determined from the equation

$$
\frac{1}{\pi} \mathscr{R} \int_{0}^{\infty} B_{i}(p) \exp \left(i p x_{1}\right) d p=g_{i}\left(x_{1}\right) \text { for } i=1,2 .
$$

The $A(p)$ in (3.4)-(3.7) is given by

$$
A(p)=\int_{0}^{a} s(t) J_{1}(p t) d t+i \int_{0}^{a} r(t) J_{0}(p t) d t,
$$

where $s(t)$ and $r(t)$ are obtained from the Fredholm equations

$$
\begin{aligned}
& r(t)+t \int_{0}^{a} K^{(0)}(u, t) r(u) d u=t \int_{-t}^{t} \frac{f_{1}(u) d u}{\left(t^{2}-u^{2}\right)^{t}} \quad \text { for } 0<t<a, \\
& s(t)+t \int_{0}^{a} K^{(1)}(u, t) s(u) d u=\int_{-t}^{t} \frac{u f_{1}(u) d u}{\left(t^{2}-u^{2}\right)^{\frac{t}{t}}} \text { for } 0<t<a,
\end{aligned}
$$

where

$$
K^{(N)}(u, t)=\int_{0}^{\infty} S(p) J_{N}(p u) J_{N}(p t) p d p
$$


In (3.16) $J_{N}$ denotes the Bessel function of order $N$, while

$$
S(p)=2 \mathscr{R}\left[\left(\lambda_{12}+\lambda_{22} \tau\right) Q(p)\right] .
$$

Finally, the $f_{1}(u)$ in (3.14) and (3.15) is defined by

$$
f_{1}(u)=f(u)+\frac{1}{\pi} \mathscr{R} \int_{0}^{\infty}\left[U(p)\left(\lambda_{12}+\lambda_{22} \tau\right)+\nabla(p)\left(\lambda_{12}+\lambda_{22} \bar{\tau}\right)\right] \exp (i p u) i p d p
$$

\section{The displacement and stress fields}

As indicated in Section 2, the displacement and stress components are written as the sum of three separate fields $u_{k}^{(p)}$ and $\sigma_{i j}^{(p)}$ for $p=1,2,3$. Now $u_{k}^{(1)}+u_{k}^{(2)}$ and. $\sigma_{i j}^{(1)}+\sigma_{i j}^{(2)}$ are given by (2.11)-(2.15) and are continuous throughout the slab. In $0<x_{2}<h u_{k}^{(3)}$ and $\sigma_{i j}^{(3)}$ are given by (2.17) and (2.18) while in $-h<x_{2}<0$ these quantities are given by (2.20) and (2.21). Hence the requirement that the stress $\sigma_{i 2}$ be continuous across $x_{2}=0$ yields

$i p\left[\sum_{\alpha}\left\{L_{i 2 \alpha} E_{\alpha}^{+}(p)-\bar{L}_{i 2 \alpha} E_{\alpha}^{-}(p)\right\}\right]+i\left[N_{i 2} A^{+}(p)+\bar{N}_{i 2} A^{-}(p)\right]-\beta_{i 2}\left[A^{+}(p)-A^{-}(p)\right]=0$.

Equation (4.1) may be rearranged to yield

$$
\sum_{\alpha} L_{i 2 \alpha} E_{\alpha}^{+}(p)+p^{-1} A^{+}(p)\left\{N_{12}+i \beta_{i 2}\right\}=\sum_{\alpha} \bar{L}_{i 2 \alpha} E_{\alpha}^{-}(p)-p^{-1} A^{-}(p)\left\{\bar{N}_{i 2}-i \beta_{i 2}\right\} .
$$

Denoting these expressions by $F_{i}(p)$ we obtain

where

$$
\begin{aligned}
& E_{\alpha}^{+}(p)=M_{\alpha i} F_{i}(p)-M_{\alpha i}\left\{N_{i 2}+i \beta_{i 2}\right\} A^{+}(p) p^{-1}, \\
& E_{\alpha}^{-}(p)=M_{\alpha i} F_{i}(p)+M_{\alpha i}\left\{N_{i 2}+i \beta_{i 2}\right\} A^{-}(p) p^{-1},
\end{aligned}
$$

$$
\sum_{\alpha} L_{i 2 \alpha} M_{\alpha j}=\delta_{i j}
$$

The displacement $u_{k}$ on $x_{2}=0$ outside the crack must be continuous and hence, from (2.17) and (2.20),

$$
\begin{gathered}
\mathscr{R} \int_{0}^{\infty}\left[\sum_{\alpha}\left\{A_{k \alpha} E_{\alpha}^{+}(p)-\bar{A}_{k \alpha} E_{\alpha}^{-}(p)\right\}+C_{k} A^{+}(p) p^{-1}+\bar{C}_{k} \bar{A}^{-}(p) p^{-1}\right] \exp \left(i p x_{1}\right) d p \\
=0 \text { for }\left|x_{1}\right|>a .
\end{gathered}
$$

Use of (4.3) and (4.4) in (4.6) yields

$$
\mathscr{R} \int_{0}^{\infty}\left[H_{k s} F_{s}(p)+R_{k}(p)\right] \exp \left(i p x_{1}\right) d p=0 \text { for }\left|x_{1}\right|>a,
$$


where

$$
\begin{aligned}
H_{k s}= & B_{k s}-\bar{B}_{k s}, \quad B_{k s}=\sum_{\alpha} A_{k \alpha} M_{\alpha s} \\
R_{k}(p)= & \left\{-B_{k i}\left(N_{i 2}+i \beta_{i 2}\right)+C_{k}\right\} A^{+}(p) p^{-1} \\
& +\left\{-\bar{B}_{k i}\left(\bar{N}_{i 2}-i \beta_{i 2}\right)+\bar{C}_{k}\right\} A^{-}(p) p^{-1}
\end{aligned}
$$

On $x_{2}=0$ over the crack the tractions $\sigma_{i 2}=P_{i}\left(x_{1}\right)$ are prescribed. Hence, from (2.9)-(2.21), we obtain

$$
\begin{aligned}
\frac{1}{\pi} \mathscr{R} \int_{0}^{\infty}\left\{F_{i}(p) i p\right. & +\sum_{\alpha}\left(L_{i 2 \alpha} E_{\alpha}^{(1)}+\bar{L}_{i 2 \alpha} E_{\alpha}^{(2)}\right) i p+\left(i N_{i 2}-\beta_{i 2}\right) A^{(1)}(p) \\
& \left.+\left(-i \bar{N}_{i 2}-\beta_{i 2}\right) A^{(2)}(p)\right\} \exp \left(i p x_{1}\right) d p=P_{i}\left(x_{1}\right) \text { for }\left|x_{1}\right|<a .
\end{aligned}
$$

Also, on $x_{2}= \pm h$ the tractions are prescribed. Hence

$$
\begin{aligned}
& \sum_{\alpha} L_{i 2 \alpha} E_{\alpha}^{(1)}(p) i p \exp \left(i p p_{\alpha} h\right)+\sum_{\alpha} L_{i 2 \alpha} E_{\alpha}^{(2)}(p) i p \exp \left(i p \tilde{p}_{\alpha} h\right) \\
&=- {\left[\sum_{\alpha} L_{i 2 \alpha} M_{\alpha j} F_{j}(p) i p \exp \left(i p p_{\alpha} h\right)-\sum_{\alpha} L_{i 2 \alpha} M_{\alpha j}\left\{N_{j 2}+i \beta_{j 2}\right\}\right.} \\
& \times A^{+}(p) i \exp \left(i p p_{\alpha} h\right)+\left(i N_{i 2}-\beta_{i 2}\right) A^{(1)}(p) \exp (i p \tau h) \\
&\left.+\left(-i \bar{N}_{i 2}-\beta_{i 2}\right) A^{(2)}(p) \exp (i p \bar{\tau} h)+\left(i N_{i 2}-\beta_{i 2}\right) A^{+}(p) \exp (i p \tau h)\right] \\
&+G_{i 1}(p),
\end{aligned}
$$

$\sum_{\alpha} L_{i 2 \alpha} E_{\alpha}^{(1)}(p) i p \exp \left(-i p p_{\alpha} h\right)+\sum_{\alpha} \bar{L}_{i 2 \alpha} E_{\alpha}^{(2)}(p) i p \exp \left(-i p \bar{p}_{\alpha} h\right)$

$$
\begin{aligned}
=- & {\left[\sum_{\alpha} L_{i 2 \alpha} \bar{M}_{\alpha j} F_{j}(p) i p \exp \left(-i p \bar{p}_{\alpha} h\right)+\sum_{\alpha} \bar{L}_{i 2 \alpha} \bar{M}_{\alpha j}\left(\bar{N}_{i 2}-i \beta_{i 2}\right)\right.} \\
& \bar{A}^{-}(p) i \exp \left(-i p p_{\alpha} h\right)+\left(i N_{i 2}-\beta_{i 2}\right) A^{(1)}(p) \exp (-i p \tau h) \\
& +\left(-i \bar{N}_{\imath 2}-\beta_{i 2}\right) \bar{A}^{(2)}(p) \exp (-i p \bar{\tau} h)+\left(-i \bar{N}_{i 2}-\beta_{i 2}\right) \\
& \left.\times A^{-}(p) \exp (-i p \bar{\tau} h)\right]+G_{i 2}(p),
\end{aligned}
$$

where

$$
\begin{aligned}
& \frac{1}{\pi} \mathscr{R} \int_{0}^{\infty} G_{i 1}(p) \exp \left(i p x_{1}\right) d p=q_{i 1}\left(x_{1}\right), \\
& \frac{1}{\pi} \mathscr{R} \int_{0}^{\infty} G_{i 2}(p) \exp \left(i p x_{1}\right) d p=q_{i 2}\left(x_{1}\right) .
\end{aligned}
$$

In (4.12) and (4.13), $q_{i 1}\left(x_{1}\right)$ and $q_{i 2}\left(x_{1}\right)$ are the specified tractions on $x_{2}=h$ and $x_{2}=-h$ respectively. 
It is convenient at this stage to introduce the matrices

$$
\begin{aligned}
R & =\left[L_{i 2 \alpha} \exp \left(-i p p_{\alpha} h\right)\right], \quad S=\left[L_{i 2 \alpha} \exp \left(i p p_{\alpha} h\right)\right], \\
M & =\left[M_{\alpha i}\right], \quad G=\left[G_{i}\right], \quad H=\left[H_{i}\right], \\
E^{(I)} & =\left[E_{\alpha}^{(I)}\right] \quad \text { for } I=1,2, \quad F=\left[F_{\alpha}\right] .
\end{aligned}
$$

Hence (4.10) and (4.11) may be written

$$
\begin{aligned}
& S E^{(1)}+\bar{R} E^{(2)}=-S M F+G, \\
& R E^{(1)}+\bar{S} E^{(2)}=-\bar{S} \bar{M} F+H,
\end{aligned}
$$

where the precise forms for $G$ and $H$ may be readily obtained by comparing (4.10) and (4.15) and also (4.11) and (4.16). Equations (4.15) and (4.16) may be used to solve for $E^{(1)}$ and $E^{(2)}$. We obtain

where

$$
\begin{aligned}
& E^{(\mathbf{1})}=Q F+X, \\
& E^{(\mathbf{2})}=Q F+Y,
\end{aligned}
$$

$$
\begin{aligned}
& Q=-\left(\bar{R}^{-1} S-\bar{S}^{-1} R\right)^{-1}\left(\bar{R}^{-1} S M-\bar{M}\right), \\
& X=\left(\bar{R}^{-1} S-\bar{S}^{-1} R\right)^{-1}\left(\bar{R}^{-1} G-\bar{S}^{-1} H\right), \\
& Y=\left(\bar{R}^{-1} S-\bar{S}^{-1} R\right)^{-1}\left(\bar{R}^{-1} \bar{H}-\bar{S}^{-1} \bar{G}\right) .
\end{aligned}
$$

Substitution of (4.17) and (4.18) into (4.9) gives

$$
\mathscr{R} \int_{0}^{\infty}\left[F_{j}(p)+T_{j k}(p) F_{k}(p)\right] i p \exp \left(i p x_{1}\right) d p=\mathscr{P}_{j}\left(x_{1}\right) \text { for }\left|x_{1}\right|<a,
$$

in which

$$
\begin{aligned}
\mathscr{P}_{j}\left(x_{1}\right)=\pi P_{j}\left(x_{1}\right) & -\mathscr{R} \int_{0}^{\infty} \sum_{\alpha}\left[L_{j 2 \alpha} X_{\alpha}(p)+L_{j 2 \alpha} \bar{Y}_{\alpha}(p)\right] i p \exp \left(i p x_{1}\right) d p \\
& -\mathscr{R} \int_{0}^{\infty}\left\{\left(i N_{j 2}-\beta_{j 2}\right) A^{(1)}(p)-\left(i N_{j 2}+\beta_{j 2}\right) A^{(2)}(p)\right\} \exp \left(i p x_{1}\right) d p
\end{aligned}
$$

and

$$
T_{j k}(p)=2 \mathscr{R} \sum_{\alpha} L_{j 2 \alpha} Q_{\alpha k}(p) .
$$

Returning to (4.7), we note that the matrix $H_{k s}$ is non-singular (see Stroh [11]) and hence there exists an inverse matrix $U_{j k}$ such that

$$
U_{j k} H_{k s}=\delta_{j s}
$$


Thus, equation (4.7) can be written as

$$
\mathscr{R} \int_{0}^{\infty}\left[F_{k}(p)+U_{k j} R_{j}(p)\right] i \exp \left(i p x_{1}\right) d p=0 \text { for }\left|x_{1}\right|>a .
$$

Now equation (4.22) may be rewritten as

$$
\begin{aligned}
\mathscr{R} \int_{0}^{\infty}\left[F_{j}(p)\right. & \left.+U_{j k} R_{k}(p)+T_{j k}(p)\left\{F_{k}(p)+U_{k r} R_{r}(p)\right\}\right] \\
& \times i p \exp \left(i p x_{1}\right) d p=\mathscr{S}_{j}\left(x_{1}\right) \text { for }\left|x_{1}\right|<a,
\end{aligned}
$$

where

$$
\mathscr{S}_{j}\left(x_{1}\right)=\mathscr{P}_{j}\left(x_{1}\right)+\mathscr{R} \int_{0}^{\infty}\left[U_{j k} R_{k}(p)+T_{j k}(p) U_{k r} R_{r}(p)\right] i p \exp \left(i p x_{1}\right) d p .
$$

Let

$$
\mathscr{F}_{k}(p)=F_{k}(p)+U_{k j} R_{j}(p) .
$$

Then (4.26) and (4.27) yield

$$
\begin{gathered}
\mathscr{R} \int_{0}^{\infty} \mathscr{F}_{k}(p) i \exp \left(i p x_{1}\right) d p=0 \text { for }\left|x_{1}\right|>a, \\
\mathscr{R} \int_{0}^{\infty}\left[\mathscr{F}_{j}(p)+T_{j k}(p) \mathscr{F}_{k}(p)\right] i p \exp \left(i p x_{1}\right) d p=\mathscr{S}_{j}\left(x_{1}\right) \text { for }\left|x_{1}\right|<a .
\end{gathered}
$$

Equation (4.30) will be satisfied if $\mathscr{F}_{k}(p)$ is taken in the form

$$
\mathscr{F}_{k}(p)=\int_{0}^{a} s_{k}(t) J_{1}(p t) d t+i \int_{0}^{a} r_{k}(t) J_{0}(p t) d t,
$$

where $s_{k}(t)$ and $r_{k}(t), k=1,2,3$, are real functions to be determined and $J_{0}$ and $J_{1}$ are Bessel functions of order zero and one respectively. Use of (4.32) in (4.31) yields

$$
\begin{gathered}
\begin{array}{c}
\int_{0}^{\infty} p \cos \left(p x_{1}\right) d p \int_{0}^{a} r_{j}(t) J_{0}(p t) d t+\int_{0}^{\infty} T_{j k}(p) p \cos \left(p x_{1}\right) d p \int_{0}^{a} r_{k}(t) J_{0}(p t) d t \\
=-\frac{1}{2}\left[\mathscr{S}_{j}\left(x_{1}\right)+\mathscr{S}_{j}\left(-x_{1}\right)\right] \text { for }\left|x_{1}\right|<a
\end{array} \\
\int_{0}^{\infty} p \sin \left(p x_{1}\right) d p \int_{0}^{a} s_{j}(t) J_{1}(p t) d t+\int_{0}^{\infty} T_{j k}(p) p \sin \left(p x_{1}\right) d p \int_{0}^{a} s_{k}(t) J_{1}(p t) d t \\
=-\frac{1}{2}\left[\mathscr{S}_{j}\left(x_{1}\right)-\mathscr{S}_{j}\left(-x_{1}\right)\right] \text { for }\left|x_{1}\right|<a .
\end{gathered}
$$


Interchanging the order of integration and using standard results for Bessel functions we obtain

$$
\begin{gathered}
\frac{d}{d x_{1}} \int_{0}^{x_{1}} \frac{r_{j}(t) d t}{\left(x_{1}^{2}-t^{2}\right)^{\frac{1}{1}}}+\int_{0}^{\infty} T_{j k}(p) p \cos \left(p x_{1}\right) d p \int_{0}^{a} r_{k}(t) J_{0}(p t) d t \\
=-\frac{1}{2}\left[\mathscr{S}_{j}\left(x_{1}\right)+\mathscr{S}_{j}\left(-x_{1}\right)\right] \quad \text { for } 0<x_{1}<a, \\
\frac{1}{x_{1}} \frac{d}{d x_{1}} \int_{0}^{x_{1}} \frac{t s_{j}(t) d t}{\left(x_{1}^{2}-t^{2}\right)^{t}}+\int_{0}^{\infty} T_{j k}(p) p \sin \left(p x_{1}\right) d p \int_{0}^{a} s_{k}(t) J_{1}(p t) d t \\
=-\frac{1}{2}\left[\mathscr{S}_{j}\left(x_{1}\right)-\mathscr{S}_{j}\left(-x_{1}\right)\right] \text { for } 0<x_{1}<a .
\end{gathered}
$$

These Abel integral equations may be inverted to yield

$$
\begin{aligned}
& r_{j}(t)+t \int_{0}^{a} K_{j k}^{(0)}(u, t) r_{k}(u) d u=-\frac{t}{\pi} \int_{-t}^{t} \frac{\mathscr{S}_{j}(u) d u}{\left(t^{2}-u^{2}\right)^{\frac{t}{2}}} \text { for } 0<t<a, \\
& s_{j}(t)+t \int_{0}^{a} K_{j k}^{(1)}(u, t) s_{k}(u) d u=-\frac{1}{\pi} \int_{-t}^{t} \frac{u \mathscr{S}_{j}(u) d u}{\left(t^{2}-u^{2}\right)^{\frac{1}{t}}} \quad \text { for } 0<t<a,
\end{aligned}
$$

where standard results for Bessel function have been used to obtain $K_{i j}^{(N)}$ in the form

$$
K_{j k}^{(N)}(u, t)=\int_{0}^{\infty} T_{j k}(p) J_{N}(p u) J_{N}(p t) p d p
$$

Equation (4.37) constitutes three simultaneous Fredholm integral equations for the $r_{j}(t)$ while (4.38) constitutes three similar equations for the $s_{j}(t)$. These equations may be solved numerically and then $\mathscr{F}_{k}(p)$ may be found through (4.32). Equations (4.29), (4.3) and (4.4) then yield $E_{\alpha}^{+}(p)$ and $E_{\alpha}^{-}(p)$ while (4.29), (4.17) and (4.18) yield $E^{(1)}(p)$ and $E^{(2)}(p)$. Hence the displacement and stress distributions throughout the slab may be calculated from (2.7)-(2.21).

\section{Numerical results}

The stress $\sigma_{i 2}\left(x_{1}, 0\right)$ near the crack tip at $r=a$ takes the form

$$
\sigma_{i 2}\left(x_{1}, 0\right) \sim K_{i} r^{-1} \text { as } r \rightarrow 0,
$$

where $r=x_{1}-a$ with $x_{1}>a$ and the $K_{i}$ are constants. In this section we use the analysis of the previous sections to determine the values of the $K_{i}$ for a particular transversely isotropic material.

For transversely isotropic materials with the $x_{1}$ and $x_{2}$ axes lying in the transverse plane, the non-zero stiffness $c_{i j k l}$, the coefficients of linear thermal expansion 
$\alpha_{i j}$, and the thermal conductivities $\lambda_{i j}$ are

$$
\begin{aligned}
& c_{1111}=c_{2222}, c_{1133}=c_{2233}, c_{1313}=c_{2323}, c_{1122}, \\
& c_{3333}, c_{1212}=\left(c_{1111}-c_{1122}\right) / 2 \\
& \alpha_{11}=\alpha_{22}, \alpha_{33}, \quad \lambda_{11}=\lambda_{22}, \lambda_{33} .
\end{aligned}
$$

If a rotation of $\alpha$ about the $x_{2}$ axis is followed by a rotation of $\theta$ about the $x_{1}$ axis, then the constants referred to the rotated frame are

$$
c_{i j k l}^{\prime}=a_{i m} a_{j n} a_{k p} a_{l q} c_{m n p q}, \quad \beta_{i j}^{\prime}=a_{i m} a_{j n} \beta_{m n}, \quad \lambda_{i j}^{\prime}=a_{i m} a_{j n} \lambda_{m n},
$$

where

$$
\left[a_{i j}\right]=\left[\begin{array}{ccc}
\cos \alpha & -\sin \alpha \sin \theta & -\sin \alpha \cos \theta \\
0 & \cos \theta & \sin \theta \\
-\sin \alpha & -\cos \alpha \sin \theta & \cos \alpha \cos \theta
\end{array}\right]
$$

For illustrative purposes we consider the constants for a crystal of zinc. Referred to symmetry axes with the $x_{3}$ axis normal to the transverse plane the constants are

$$
\begin{aligned}
& c_{1111}=16.5, \quad c_{1122}=3.1, \quad c_{1133}=5, \quad c_{3333}=6.2, \\
& c_{1313}=3.92, \quad 10^{6} \alpha_{11}=60.8, \quad 10^{6} \alpha_{33}=14.3, \quad \lambda_{11} / \lambda_{33}=1.17 .
\end{aligned}
$$

If the elastic constants are multiplied by $10^{11}$ then the units for these constants are dynes $/ \mathrm{cm}^{2}$, while the coefficients of thermal expansion are for a temperature increase of one degree centigrade.

Consider a slab with $h / a=20$. Further let $a=1$ and suppose the crack faces and slab faces $x_{2}= \pm h$ are traction free. Also suppose the temperature on the slab faces is zero while over the crack the heat flux is constant so that, in (3.1),

$$
f\left(x_{1}\right)=f_{0},
$$

where $f_{0}$ is a constant. The values of $K_{i}$ for various combinations of the angles $\alpha$ and $\theta$ are given in Table 1 . The quantity $K_{0}$ in the table is $K_{1}$ calculated for $\alpha=0$ and $\theta=\pi / 2$.

TABLE 1

\begin{tabular}{llllrrl}
\hline$\alpha$ & 0 & 0 & $\pi / 4$ & $\pi / 4$ & $\pi / 3$ & $\pi / 2$ \\
$\theta$ & $\pi / 2$ & $\pi / 4$ & 0 & $\pi / 4$ & $\pi / 6$ & 0 \\
$K_{1} / K_{0}$ & 1 & 1.46 & 1.19 & -1.72 & -1.52 & 0.85 \\
$K_{2} / K_{0}$ & 0 & 0 & 0 & 1.35 & 1.22 & 0 \\
$K_{\mathrm{s}} / K_{0}$ & 0 & 0 & -2.02 & 0.85 & 1.12 & 0 \\
\hline
\end{tabular}


When $\alpha=0$ and $\theta=\pi / 2$, each of the planes $x_{i}=0, i=1,2,3$, is a plane of elastic symmetry and it is clear from Table 1 and equation (5.1) that, in this case, the stress $\sigma_{12}$ is singular in the plane of the crack but $\sigma_{22}$ and $\sigma_{32}$ do not exhibit singular behaviour.

When $\alpha=0$ and $\theta=\pi / 4$, the $x_{1}=0$ plane is a plane of elastic symmetry but the $x_{2}=0$ and $x_{3}=0$ planes are not planes of the crack is similar to the above case when $\alpha=0$ and $\theta=\pi / 2$.

When $\alpha=\pi / 4$ and $\theta=0$, the $x_{2}=0$ plane is a plane of elastic symmetry while the $x_{1}=0$ and $x_{3}=0$ planes are not planes of elastic symmetry. In this case both $\sigma_{12}$ and $\sigma_{32}$ exhibit singular behaviour in the plane of the crack while $\sigma_{22}$ does not exhibit singular behaviour.

When $\alpha=\pi / 4$ and $\theta=\pi / 4$, or $\alpha=\pi / 3$ and $\theta=\pi / 6$, none of the planes $x_{i}=0$, $i=1,2,3$, are planes of elastic symmetry and all the stresses $\sigma_{i 2}, i=1,2,3$, exhibit singular behaviour.

When $\alpha=\pi / 2$ and $\theta=0$, each of the planes $x_{i}=0, i=1,2,3$, is a plane of elastic symmetry and the singular behaviour of the stress in the plane of the crack is similar to the first case considered above when $\alpha=0$ and $\theta=\pi / 2$.

It is of interest to compare these results with those obtained by Sih [9] for the thermal stress singularity at the tip of a crack in an isotropic material. Sih's results show that a constant heat flux over the crack face gives rise to a singular stress $\sigma_{12}$ in the plane of the crack while the stresses $\sigma_{22}$ and $\sigma_{23}$ remain non-singular. The results obtained here indicate that this situation also holds true for anisotropic material provided the $x_{1}=0$ plane is a plane of elastic symmetry. However, if the $x_{1}=0$ plane is not a plane of elastic symmetry, then at least one of the stresses $\sigma_{22}$ and $\sigma_{23}$ is singular in the plane of the crack.

Finally, we note that the displacements induced by purely mechanical loads applied to the surfaces of the anisotropic slab with the crack stress-free have been studied in detail in a previous paper (Clements and Tauchert [7]). An analysis of temperature-induced displacements can be carried out in a similar fashion using the present formulation.

\section{References}

[1] A. Y. Aköz and T. R. Tauchert, "Thermal stresses in an orthotropic elastic semispace", J. Appl. Mech. 39 (1972), 87-90.

[2] C. Atkinson and D. L. Clements, "On some crack problems in anisotropic thermoelasticity", Int. J. Solids Structs. 13 (1977), 855-864.

[3] Y.P. Chang, "Analytical solution for heat conduction in an anisotropic media in infinite, semi-infinite and two-plane-bounded region", Int. J. Heat Mass Transfer 20 (1977), 1019-1028. 
[4] D. L. Clements, "A crack in an anisotropic elastic slab", Quart. Appl. Math. 4 (1977), 437-443.

[5] D. L. Clements, "A crack in an anisotropic layered material", Engng. Trans. (to appear).

[6] D. L. Clements, "Thermal stress in an anisotropic elastic half-space", SIAM J. Appl. Math. 24 (1973), 332-337.

[7] D. L. Clements and T. R. Tauchert, "Deformations of an anisotropic elastic slab containing a crack", Acta Mechanica (to appear).

[8] D. L. Clements and G. D. Toy, "Two contact problems in anisotropic thermoelasticity", J. Elasticity 6 (1976), 137-147.

[9] G. C. Sih, "On the singular character of thermal stresses near a crack tip", J. Appl. Mech. 29 (1962), 587-589.

[10] A. J. M. Spencer, Deformations of fibre-reinforced materials (O.U.P., 1972).

[11] A. N. Stroh, "Dislocations and cracks in anisotropic elasticity", Phil. Mag. 3 (1958), 625-646.

[12] T. R. Tauchert, "A review: quasistatic thermal stresses in anisotropic elastic bodies, with applications to composite materials", Acta Mechanica 23 (1975), 113-135.

[13] T. R. Tauchert and A. Y. Aköz, "Stationary temperature and stress fields in an anisotropic elastic slab", J. Appl. Mech. 42 (1975), 647-650.

Department of Applied Mathematics

University of Adelaide

Adelaide, S.A. 5000

Australia

and

Department of Engineering Mechanics

University of Kentucky

Lexington, Kentucky

U.S.A. 